\title{
Wie viel Lärm ist zu viel?
}

\section{Ottilia Lütolf Elsener}

Fachärztin Angiologie und Innere Medizin, Mitglied Ärztinnen und Ärzte für Umweltschutz (AefU), Mitglied Eidgenössische Kommission für Lärmbekämpfung (EKLB), Arbeitsgruppe «Tag gegen Lärm», Mitglied FMH

Ecoplanstudie im Auftrag des BAFU «Auswirkungen des Verkehrslärms auf die Gesundheit», Bern und Altdorf, 2014

2 Lärmwirkungsstudie NORAH, Bochum, 2015 Christensen JS, Hjortebjerg D, Raaschou-Nielsen O, Ketzel M, Sørensen TI, Sørensen M. Pregnancy and childhood exposure to residential traffic noise and overweight at 7 years of age. Environment International. 2016;94:170-6.

\footnotetext{
Der Artikel entstand in Zusammenarbeit mit Nadia Sauter Oes, Fachärztin FMH für Kinder- u. Jugendmedizin, Kinderärzte Schweiz, Winterthur, und Andrea Kaufmann, Koordinationsstelle «Tag gegen Lärm», Luzern.
}

Ob Schlafstörungen, erhöhter Blutdruck oder Hörschäden - Lärm kann Menschen krank machen. Doch nicht nur Erwachsene leiden unter Lärm, auch Kinder sind davon betroffen. Die Folgen einer übermässigen Lärmexposition sind aber nicht nur körperlicher Natur, auch psychische Leiden oder eine Beeinträchtigung der kognitiven Leistungen können auftreten.

Lärm ist ein Stressor. Unser Gehör ist als hochsensibles Organ an die Geräuschkulisse einer längst vergangenen Naturlandschaft angepasst. Laute und ungewohnte Geräusche waren ursprünglich ein Signal für Gefahr, auf die der menschliche Körper mit einer erhöhten Alarmbereitschaft zu Flucht oder Kampf reagierte. Unsere Geräuschkulisse hat sich dramatisch verändert: Eine Unmenge von verschiedensten Geräuschen überflutet uns ständig. Auf laute und störende Geräusche reagiert unser Körper aber immer noch gleich wie zu Urzeiten. Er schüttet vermehrt Adrenalin und Noradrenalin aus und setzt Energiereserven frei. Mit steigender Lautstärke und Dauer gesellt sich Cortisol dazu mit Folgen für unser Wohlbefinden und unsere Gesundheit. Eine Gewöhnung des Körpers an Lärm gibt es nicht.

\section{Auch Kinder leiden unter Lärm}

Die Wirkung von Lärm auf Kinder ist selten Gegenstand von medialen Diskussionen, doch Studien dokumentieren, dass chronische Lärmbelastungen auch bei Kindern anhaltende seelische und körperliche Beeinträchtigungen auslösen können. Wir müssen uns bewusst sein, dass Kinder ihre akustische Umwelt weni-

\section{Tag gegen Lärm}

Am 26. April 2017 findet der Internationale Tag gegen Lärm statt. Seit 2005 nimmt die Schweiz an diesem Aktionstag teil. Jedes Jahr steht eine andere Facette der Lärmproblematik im Vordergrund. Dieses Jahr wird unter dem Motto «Ruhe fördert» auf die Auswirkungen von Lärm auf Kinder aufmerksam gemacht. Lärm stört und kann krank machen. Für eine gesunde Entwicklung brauchen Kinder eine gesunde Umgebung - ganz im Sinne von «Ruhe fördert»! Um Eltern und Betroffene auf dieses wichtige Thema aufmerksam zu machen, stellen wir Ihnen attraktive Flyer, die ausgeschnitten einen Türhänger ergeben, zum Auflegen in Ihrer Praxis zur Verfügung. Bestellen Sie noch heute kostenlos unter www.lärm.ch/kinder und helfen Sie mit, Kinder von übermässigem Lärm und dessen schädlichen Folgen zu schützen. DieTrägerschaft des "Tag gegen Lärm» in der Schweiz setzt sich zusammen aus:

Cercle Bruit (Vereinigung kantonaler Lärmfachstellen), Schweizerische Gesellschaft für Akustik, Ärztinnen und Ärzte für Umweltschutz. Unterstützt wird dieTrägerschaft vom Bundesamt für Umwelt BAFU und vom Bundesamt für Gesundheit BAG. Weitere Informationen finden Sie unter www.lärm.ch.

ger beeinflussen können als Erwachsene. Sie sind oft Lärm ausgesetzt, ohne diesem ausweichen zu können. Ihre altersentsprechenden Bewältigungsstrategien (sog. Coping) schützen sie nicht vor den gesundheitlichen Folgen des Lärms. Zudem fehlen Kindern oft das Wissen und das Verständnis dafür, dass Lärm schädlich sein kann.

\section{Lernschwierigkeiten}

Gemäss der Ecoplanstudie ${ }^{1}$ von 2014 entwickeln 17,5 Prozent aller Kinder zwischen 7 und 19 Jahren Lernschwierigkeiten, weil sie Verkehrslärm ausgesetzt sind. Der Lärm beeinträchtigt das Gedächtnis der Kinder. Dies führt zu einer eingeschränkten Konzentrationsund Merkfähigkeit, was den Lernprozess im Vergleich zu nicht lärmexponierten Kindern verlangsamt.

Im Oktober 2015 wurden die Ergebnisse der NORAHStudie $^{2}$ veröffentlicht. Dabei wurde die langfristige Wirkung von Verkehrslärm auf Gesundheit, Lebensqualität und die kindliche Entwicklung im RheinMain-Gebiet untersucht. In stark von Fluglärm belasteten Gebieten lernen Grundschulkinder langsamer lesen als Kinder in ruhigen Lagen. So verzögert eine Lärmzunahme von 10 Dezibel das Lesenlernen um einen Monat.

Leiden die kognitiven Leistungen der Kinder unter einer starken Lärmexposition, wirkt sich das auch auf ihr Erwachsenenleben aus. Mit einem verminderten Lese-, Schreib- oder Merkvermögen werden sie es in der höheren schulischen Ausbildung schwerer haben.

\section{Schlafstörungen und Verhaltens- auffälligkeiten}

Lärm wirkt sich aber nicht nur auf die kognitiven Leistungen der Kinder aus. Insbesondere Schlafstörungen und Stress durch Strassenlärm können auch Übergewicht und Diabetes als Folge haben. ${ }^{3}$ Aufwachreaktionen während der Nacht laufen oft unbewusst ab, das heisst, am Morgen wissen die Kinder nicht, dass ihr Schlaf gestört war. Kaum ein Kind sagt, es habe schlecht geschlafen. Dabei ist die nächtliche Erholung gerade für Kinder immens wichtig. Schlafstörungen bleiben so oft unbemerkt und beeinträchtigen über längere Zeit eine gesunde Entwicklung. 
Kinder, deren Zimmer zu einer viel befahrenen Strasse hinaus liegt, tendieren auch zu einem leicht erhöhten Blutdruck. Leidet der Körper jahrelang unter Bluthochdruck, kann die Häufigkeit von kardiovaskulären Erkrankungen im Alter zunehmen. Hyperaktivität und andere Verhaltensauffälligkeiten werden ebenfalls mit einer andauernden Strassenlärmexposition in Verbindung gebracht. ${ }^{4}$

Auch in vermeintlich ruhigeren, ländlichen Gebieten leiden Kinder unter dem Lärm. Eine Untersuchung ${ }^{5}$ an 1280 Tiroler Volksschülerinnen und -schülern zeigte, dass Symptome wie Angst, Depression oder Verhaltensauffälligkeiten signifikant mit einer höheren Verkehrslärmbelastung im Alltag einhergehen. Es scheint, dass alpine Täler weniger Belastungen durch Schallquellen ertragen, da der menschliche Organismus relativ zu seiner Hintergrundbelastung reagiert. Die hohe nächtliche Spitzenschallbelastung durch den Schienenverkehr fiel in dieser Untersuchung besonders ins Gewicht.

\section{Einfluss von Lärm im Säuglingsalter}

Lärm hinterlässt Spuren im Gehirn. Die US-Forscher Edward Chang und Michael M. Merzenich ${ }^{6}$ beschallten neugeborene Ratten mehrere Monate lang mit einem Rauschen, das sich mit normalen Umweltgeräuschen im Alltag vergleichen lässt. Das verursachte zwar keine direkten Schäden im Gehör der Tierbabys, doch es wurde eine Verzögerung der Entwicklung der Hörrinde beobachtet. Dort bilden sich während des ersten Lebensmonats Neuronenverbände, die selektiv auf bestimmte Lautmuster und Frequenzen reagieren. Bei den Ratten, die der Dauerbeschallung ausgesetzt waren, blieb dieser Reifungsprozess noch nach Monaten aus. Es ist denkbar, dass ähnliche Umstände auch bei Babys nachhaltige Spuren hinterlassen. So können laute Hintergrundgeräusche im Säuglingsalter einerseits die Gehirnentwicklung beeinflussen und so die Hör- und Sprachfähigkeit beeinträchtigen. Andererseits lernen Kleinkinder weniger gut sprechen, wenn beispielsweise Radio und Fernseher ständig nebenher laufen und sie dadurch auch weniger Ansprache erhalten. Es fällt den Kleinkindern schwer, an sie gerichtete Worte aus dem Umgebungslärm herauszufiltern doch sprechen lernen Kinder nun mal durch die direkte Ansprache und das Zuhören.

\section{Hörschäden}

Verschiedene Untersuchungen bei Kindern und Jugendlichen zeigen, dass eine Beschallung mit 100 bis $120 \mathrm{~dB}(\mathrm{~A})$ über Minuten bis Stunden zu einem akuten
Lärmschaden führen kann. Eine solche Schallbelastung führt sowohl zu stoffwechselbedingten als auch mechanischen Schäden im Bereich der Hörsinneszellen des Innenohrs. Meist entwickeln sich Hörschäden schleichend, in der Kindheit und Jugend oft unbemerkt. Bei einem Schalldruck von $130 \mathrm{~dB}(\mathrm{~A})$ reichen aber bereits einige Sekunden, um einen Hörschaden zu erleiden.

Bei einem Säugling bis zum achten Lebensmonat sind die Nerven der Hörbahn von der Gehörschnecke zum Gehirn noch nicht ausgereift. Eine 15- bis 30-minütige unmittelbare Guggenmusik-Exposition beispielsweise kann da bereits zu einer irreversiblen Schädigung des Hörvermögens führen. Erwachsene und ältere Kinder können sich in solchen Fällen durch das Zuhalten der Ohren schützen, oder sie entfernen sich von der Lärmquelle. Kleinkinder und Säuglinge können das nicht. Auch anatomische Besonderheiten beim kindlichen Gehörgang führen dazu, dass die gleiche Lautstärke beim Kind einen deutlich lauteren Höreindruck verursacht als beim Erwachsenen. So kann bereits nach deutlich kürzerer Lärmexposition ein Hörschaden eintreten. Bis zum 5. Geburtstag ist das kindliche Gehör besonders empfindlich.

\section{Gesunde Entwicklung}

Damit sich Kinder möglichst gesund entwickeln können, ohne dass sie negative Langzeitfolgen davontragen, benötigen sie eine «gesunde» Umgebung. Der Belastung durch Lärm muss in Zukunft mehr Aufmerksamkeit geschenkt werden. Denn eine übermässige Lärmexposition im Säuglings- und Kindesalter kann lebenslange Folgen nach sich ziehen. Bereits 1910 erkannte Robert Koch, Bakteriologe: «Eines Tages wird der Mensch den Lärm ebenso unerbittlich bekämpfen müssen wie die Cholera und die Pest.» Seine Prophezeiung hat uns bereits eingeholt.

\section{Lärm und Lautstärke}

Lärm ist unerwünschter Schall. Die Lautstärke von Schall wird in Dezibel dB angegeben. Die logarithmische Dezibelskala bildet von $0 \mathrm{~dB}$ (Hörschwelle) bis ca. $130 \mathrm{~dB}$ (Schmerzgrenze) den gesamten Lautstärkebereich in überschaubaren Schritten ab; $10 \mathrm{~dB}$ : Ticken einer Armbanduhr, 40 dB: leise Musik, 60 dB: normale Unterhaltung, $90 \mathrm{~dB}$ : Lastwagen, $120 \mathrm{~dB}$ : Presslufthammer.

Änderungen der Lautstärke um $1 \mathrm{~dB}$ kann der Mensch unter bestimmten Voraussetzungen wahrnehmen; eine Änderung um 10 $\mathrm{dB}$ entspricht etwa einer Verdopplung bzw. Halbierung der subjektiv empfundenen Lautstärke. 